\title{
Robust Motion Control of Industrial Robot Based on Robot Parameter Identification and Feedforward Control Considering Resonant Frequency
}

\author{
Somsawas Tungpataratanawong* Student Member \\ Kiyoshi Ohishi* ${ }^{*}$ Member \\ Toshimasa Miyazaki** Member
}

In control application of industrial robot manipulator, a two-inertia resonant system is normally represented as the basic plant model for various control schemes. Various control techniques with partially feedback linearization have been proposed to achieve high performance motion control. The design of such controllers basically relies on system mechanical parameters. The proper parameters of the model cannot be obtained by the parameter identification based on only manipulator force and motion measurements. In this paper, the open-loop resonant frequency characteristic of the flexible joint is employed to identify the proper mechanical parameters of the two-inertia model. The nominal link inertia and spring constant of gear drive can be readily measured by this novel identification method.

The identified parameters are used in independent-joint controller design for conventional PD control scheme and robust control scheme to verify the effectiveness of the proposed identification method. Moreover, the accuracy improvement of the proposed robust control scheme based on feedforward inverse dynamic compensation and D-PD position control gives support the validity of the proposed identification method.

Keywords: D-PD position control, feedforward inverse dynamic compensation, parameter identification, resonant frequency, twoinertia system

\section{Introduction}

Industrial robot manipulator plays an important role in growing industry. Light weight and high load-to-weight ratio construction is required in present robots, in order to fit the fast motion and high efficiency in operation. The compact high-gear reduction with small backlash, such as planetary gear, is frequently used for the driving system in robotic applications ${ }^{(1)}$. Demandingly, the servo system must achieve the fast speed response and precise positioning at the same time. Such gear and coupling which are used as an essential part in power transmission cause the flexibility in robot's joint. The elasticity in coupling components of the gear drive system causes not only the decrement in stiffness but also the performance of the servo system in comparison with the rigid-joint robot.

Many control laws that are well applied to rigid robots ${ }^{(2)}$ have been reviewed and simple controllers have been developed for flexible joint control ${ }^{(3)(4)}$. Controllers are designed based on mechanical parameters of the elastic joint robot that can be modeled as a two-inertia mechanical structure ${ }^{(5)-(7)}$ in Fig. 1, where $\theta_{M}$ is angular position of driving motor, $\theta_{L}$ is angular position of link, $\theta_{S}$ is torsional angular position, $K_{S}$ is elastic constant of joint, and $R_{g}$ is gear ratio $\left(R_{g}>1\right)$. The rigid link and rotor inertias are denoted by $J_{L}$ and $J_{M}$, respectively. The controller design is dependent on these mechanical parameters. The parameter identification techniques

\footnotetext{
* Nagaoka University of Technology

1603-1, Kamitomioka-cho, Nagaoka 940-2188

** Nagaoka National College of Technology

888, Nishikatakai, Nagaoka 940-8532
}

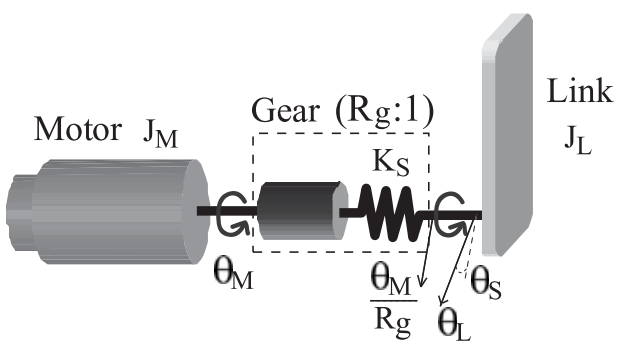

Fig. 1. Mechanical model of flexible joint

based on manipulator force and motion measurements have appeared in the literature ${ }^{(8)-(10)}$. However, these techniques are time-consuming processes and require different special test motions depending on the complex structure of the robot dynamics. Moreover, the elastic constant of joint cannot be identified by these methods.

This paper proposes a new identification method based on mechanical resonance is proposed. This method provides simple and fine determination of the nominal parameters of the two-inertia model. The identification technique utilizes the resonant and anti-resonant characteristic of each joint to estimate the nominal link inertia and elastic constant of the flexible joint.

Moreover, this paper proposes a new robust control scheme based on feedforeward inverse dynamic compensation and DPD position control ${ }^{(7)}$.

\section{Conventional Joint Motion Control for Indus- trial Robot}

In this paper, the mathematical model of each flexible joint 


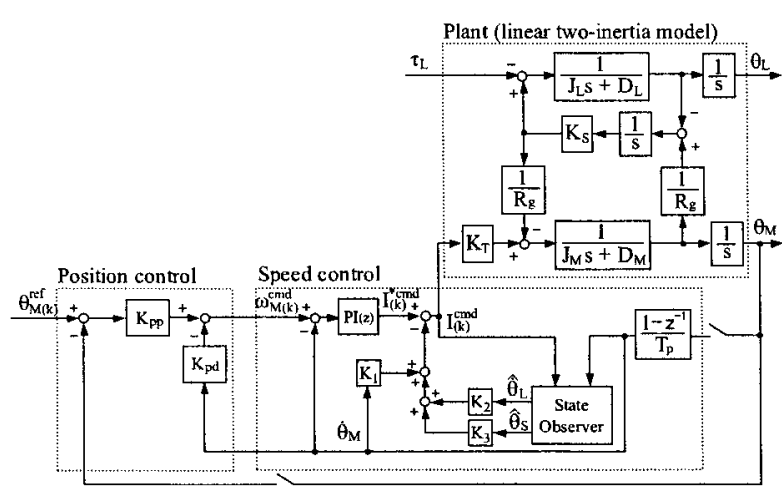

Fig. 2. Conventional joint motion control scheme (Controller1) for industrial robot

is regarded as two independent inertias, robot link inertia $J_{L}$ and motor inertia $J_{M}$, which is coupled by a linear torsional spring with stiffness $K_{S}$, as shown in Fig. 1. Damping coefficients of the link and motor are denoted by $D_{L}$ and $D_{M}$, respectively. These damping coefficients represent the viscous friction in the rotor and the coupling gear. The torque required for driving each joint is $\tau$ that relates to the command current of motor $I^{c m d}$ by torque constant $K_{T}$. The coupled torque $T_{L}$ from other links is considered as a disturbance torque to the system. The PD position controller has been proved to be applicable to elastic-joint manipulators ${ }^{(4)}$. The conventional PD position controller including PI speed control with state feedback is used as a conventional control scheme for trajectory tracking control. This conventional scheme is illustrated by block diagram in Fig. 2 and will be denoted by Controllerl hereafter.

The vibration induced by flexibility of the joint is suppressed by state feedback and PI controller in speed control loop. The PD controller is employed to stabilize the desired reference position $\theta_{M}^{r e f}$ with proportional gain $K_{p p}$ and derivative gain $K_{p d}$.

The dynamic model of robot is given in joint-space by Lagrange-Euler equations ${ }^{(11)}$, where $\tau$ is the generalized $n$ torque input vector. The matrix $M(\theta)$ is an $n \times n$ symmetric positive-definite mass matrix, $V(\theta, \dot{\theta})$ is an $n$ vector of centrifugal and Coriolis terms, $G(\theta)$ is an $n$ vector of gravity terms, and $F\left(\dot{\theta}_{L}\right)$ is viscous friction terms.

$$
\tau=D\left(\theta_{L}\right) \ddot{\theta}_{L}+V\left(\theta_{L}, \dot{\theta}_{L}\right)+G\left(\theta_{L}\right)+F\left(\dot{\theta}_{L}\right) . \cdots \cdots
$$

The only nominal terms of link inertia and viscous friction are used in two-inertia model parameters. Other terms are regarded as disturbance of the system. These nominal terms are necessary in controller design and the control performance relies on these parameters. The parameter identification techniques based on manipulator force and motion measurements ${ }^{(8)-(10)}$ are generally divided into two steps of motion test:

- constant speed test, and

- constant acceleration test.

The drawback of this identification method is that the different special motions depending on the complex structure of robot dynamics are necessary in identification process. In addition, the elastic constant of the gear drive cannot be identified experimentally by this method. Therefore, the conventional model based PD controller does not earn a good
Table 1. Nominal robot arm parameters from conventional identification method

\begin{tabular}{|c|c|c|c|c|}
\hline \multirow[b]{2}{*}{$\begin{array}{l}\text { Manipulator } \\
\text { joint No. }\end{array}$} & \multirow{2}{*}{$\begin{array}{c}\text { Identified parameters } \\
J_{L} \\
\left(\mathrm{kgm}^{2}\right)\end{array}$} & \multicolumn{3}{|c|}{ Known parameters } \\
\hline & & $\begin{array}{c}K_{S} \\
(\mathrm{Nm} / \mathrm{rad})\end{array}$ & $\begin{array}{c}J_{M} \\
\left(\mathrm{kgm}^{2}\right)\end{array}$ & $R_{g}$ \\
\hline Jt1 & 14.34 & 67000 & $1.2 \times 10^{-3}$ & 100 \\
\hline $\mathrm{Jt} 2$ & 49.50 & 180000 & $3.4 \times 10^{-4}$ & 160 \\
\hline $\mathrm{Jt} 3$ & 13.0 & 60000 & $2.5 \times 10^{-5}$ & 160 \\
\hline
\end{tabular}

position control response for flexible joint robot.

The only nominal link inertia is explicitly identified from motion test and used in two-inertia model. Other necessary parameters for two-inertia model are not actually obtained from this motion test. Hence, the accuracy of the identified parameters is not guaranteed for the conventional identification method. Especially, the deviation in elastic constant of the gear can deteriorate the response of the control system. Table 1 shows the identified link inertia based on the known values of motor inertia and elastic constant of the gear.

\section{Parameter Identification in Frequency Do- main}

With consideration of two-inertia characteristic in each joint, the frequency response of each robot arm identifies both the link inertia $\left(J_{L}\right)$ and the elastic constant $\left(K_{S}\right)$ of the coupling gear. The equations for the two-inertia flexible model are expressed in

$$
\begin{aligned}
& J_{M} \ddot{\theta}_{M}+D_{M} \dot{\theta}_{M}+\frac{K_{S}}{R_{g}} \theta_{S}=\tau, \cdots \ldots \ldots \ldots \ldots \ldots \ldots \ldots \ldots \ldots \ldots \ldots \ldots \ldots \\
& J_{L} \ddot{\theta}_{L}+D_{L} \dot{\theta}_{L}-K_{S} \theta_{S}=0 . \cdots \cdots \cdots \cdots \cdots \cdots \cdots
\end{aligned}
$$

In parameter identification experiment, motor speed is used as the output of the system on the assumption that the effects of damping coefficients $\left(D_{M}\right.$ and $\left.D_{L}\right)$ are negligible. The relationship from the current command to the motor speed gives the characteristic of mechanical resonant frequency $\omega_{r}$, and anti-resonant $\omega_{a r}$. The mechanical resonant and anti-resonant frequency can be shown by two-inertia parameters as

$$
\begin{aligned}
& \omega_{r}=\sqrt{\frac{K_{S}}{J_{L}}+\frac{K_{S}}{J_{M} R_{g}^{2}}}, \cdots \\
& \omega_{a r}=\sqrt{\frac{K_{S}}{J_{L}}} \cdots \cdots \cdots
\end{aligned}
$$

When the $\omega_{r}$ and $\omega_{a r}$ are obtained, the nominal values of link inertia and elastic constant is identified on the assumption that the motor inertia and gear ratio are already known as shown by Eq. 6 and 7. This paper utilizes the physically parameterized model in frequency domain on the assumption that motor inertia is already known. By investigating the frequency response of the two-inertia system, the nominal link inertia and the elastic constant, are identified in accordance with the resonant and anti-resonant point of the system characteristic.

$$
\begin{aligned}
J_{L} & =\left(\frac{\omega_{r}^{2}}{\omega_{a r}^{2}}-1\right) J_{M} R_{g}^{2} \ldots \ldots \ldots \ldots \ldots \ldots \ldots \ldots \ldots \ldots \ldots \ldots \ldots \ldots \ldots \ldots
\end{aligned}
$$


The new parameter identification method explicitly obtains more accurate parameters in comparison with the conventional method, which assumes the known values of elastic constant of the gear. Normally, the deviation of elasticity in gear drive has more influence than the deviation of motor inertia. Therefore, the improper value of elastic constant may substantially degrade the performance of the control system.

The identification experimental system is the open loop system without any state feedback and motor position. The motor position response is observed during the motion under swept sine command current. The parameter identification experiment is depicted in Fig. 3.

The motor driver system contains a fast current control loop for motor torque control. The data collection for parameter identification of the robot is obtained by measuring the input signal $I^{c m d}$ and the output signal $\theta_{M}$ including the reconstructed motor speed.

The frequency response from the experiment is then plotted and examined for the resonant and anti-resonant frequency. Bode plot for the identified model of the second joint (Jt2) of the experimental setup robot is shown in Fig. 4. This bode plot corresponds to the two-inertia flexible model with input $I^{c m d}$ and output $\dot{\theta}_{M}$. The resonant and anti-resonant frequencies of all three joints are obtained and shown in Table 2 with the known values of motor inertia and gear ratio.

To show the control performance of these identification methods, the trajectory tracking control is implemented by Controllerl on the planar circle reference path. The

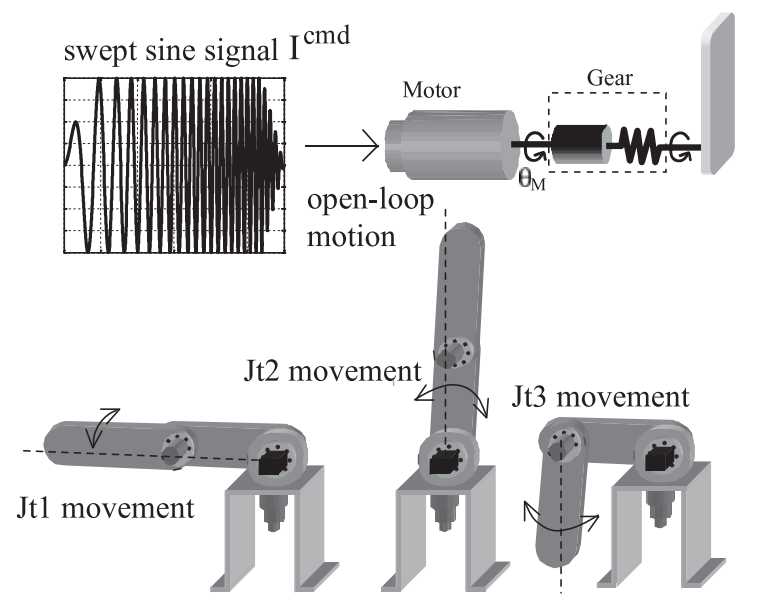

Fig. 3. Parameter identification experiment
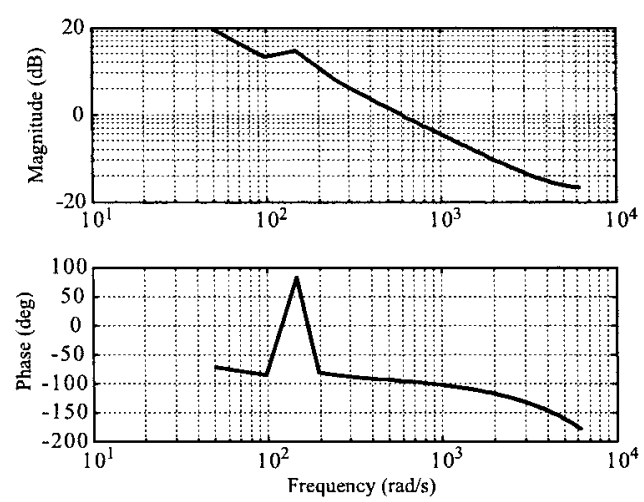

Fig. 4. Frequency characteristic from identification experiment of $\mathrm{Jt} 2$ movements of the experimental robot are executed as illustrated in Fig. 5. The robot is driven with trajectory velocity at $0.25(\mathrm{~m} / \mathrm{s})$ for low speed motion and $0.5(\mathrm{~m} / \mathrm{s})$ for high speed motion. The high speed motion is realized by accelerating the actuator beyond the rated speed to reach $85 \%$ of its maximum speed. For low speed, actuator is driven up to $40 \%$ of the maximum speed. The radius variation of the measured tracking path $|\Delta r|$ is evaluated through the trajectory as shown in Fig. 6. The control performance is investigated based on $|\Delta r|$ of the tracking path. Fig. 7 and 8 show the circle trajectory tracking response of Controllerl designed by the parameters based on the conventional identification method in comparison with the parameters based on the proposed identification method. It is obvious that to improve the performance of the control system, link inertias and elastic constants should be identified and used as the nominal parameters in speed and position controller design. The proposed parameter identification technique realizes the improvement of conventional controller design. However, nonlinear effects should be eliminated in order to achieve the quick response in motion control. The robust control scheme is therefore demanded to implement on the robot to improving the overall performance of the motion control.

Table 2. Resonant and anti-resonant frequencies with identified parameters for two-inertia flexible joint model

\begin{tabular}{c|c|c|c|c|c|c}
\hline \hline \multirow{2}{*}{$\begin{array}{c}\text { Manipulator } \\
\text { joint No. }\end{array}$} & \multicolumn{4}{|c|}{$\begin{array}{c}\text { Identified parameters } \\
\text { from proposed method }\end{array}$} & \multicolumn{2}{c}{ Known parameters } \\
\cline { 2 - 7 } & $\begin{array}{c}\omega_{r} \\
(\mathrm{rad} / \mathrm{s})\end{array}$ & $\begin{array}{c}\omega_{a r} \\
(\mathrm{rad} / \mathrm{s})\end{array}$ & $\begin{array}{c}J_{L} \\
\left(\mathrm{kgm}^{2}\right)\end{array}$ & $\begin{array}{c}K_{S} \\
(\mathrm{Nm} / \mathrm{rad})\end{array}$ & $\begin{array}{c}J_{M} \\
\left(\mathrm{kgm}^{2}\right)\end{array}$ & $R_{g}$ \\
\hline Jt1 & 129 & 105 & 6.0 & 67000 & $1.2 \times 10^{-3}$ & 100 \\
$\mathrm{Jt} 2$ & 147 & 98 & 10.88 & 105000 & $3.4 \times 10^{-4}$ & 160 \\
Jt3 & 330 & 220 & 0.8 & 38720 & $2.5 \times 10^{-5}$ & 160 \\
\hline \hline
\end{tabular}

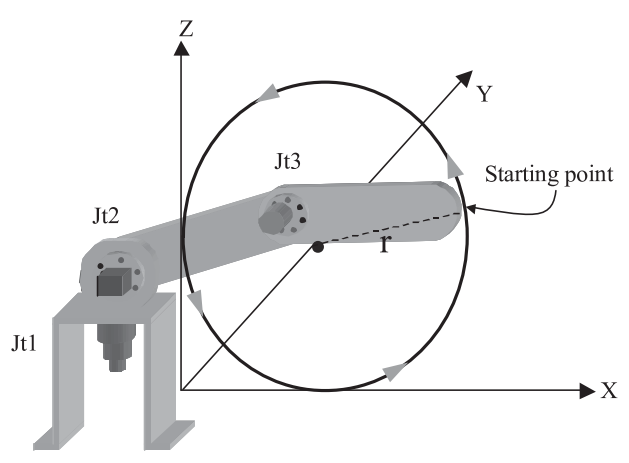

Fig. 5. Circle trajectory tracking experiment

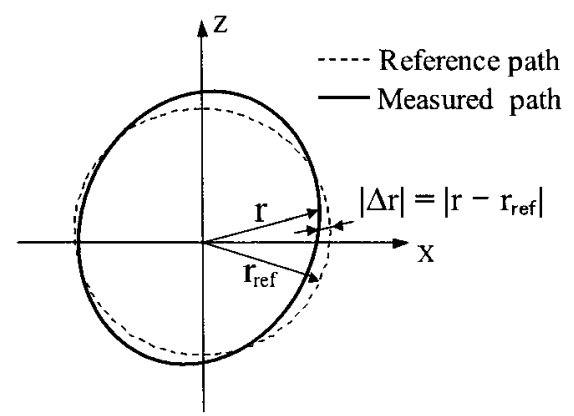

Fig. 6. Evaluation of circle trajectory tracking 


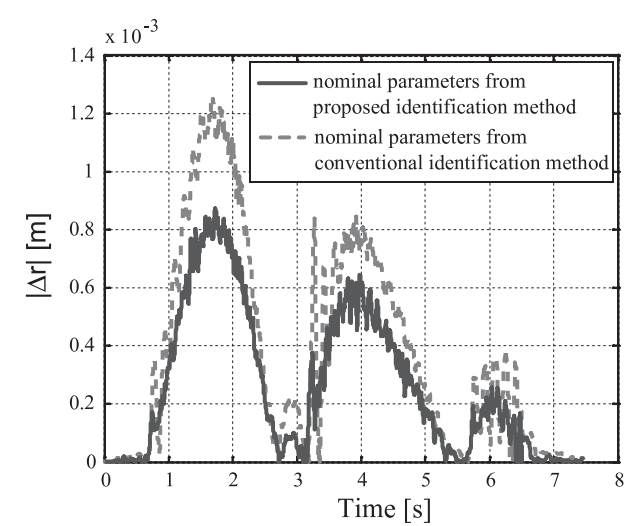

Fig. 7. Comparison of low-speed tracking path errors in conventional control scheme

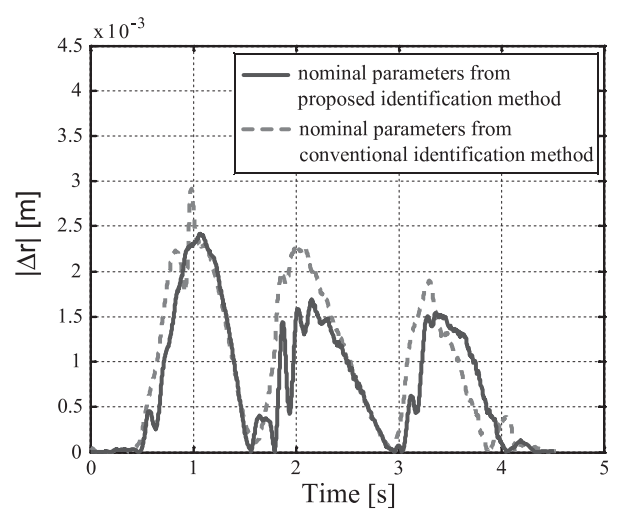

Fig. 8. Comparison of high-speed tracking path errors in conventional control scheme

\section{Robust Motion Control Scheme}

The robust motion control scheme is introduced to cope with parameter variation and coupled torque from other joints while the manipulator is moving. In addition, the gravity compensation in feedforward manner is also included in the robust motion control scheme. This robust control scheme, which is denoted by Controller2, comprises feedforward inverse-dynamic torque compensation and two-degreeof-freedom D-PD position control with robust pole assignment and feedforward pole cancellation in speed control. The control scheme block diagram is shown in Fig. 9. The Controller 2 scheme is also designed corresponding to the twoinertia model of flexible-joint robot.

4.1 Robust Speed Control The improvement of PI and state feedback in inner speed control loop of the Controllerl, is to employ the feedforward controller to eliminate the dominant complex conjugate closed-loop poles of the inner control loop which depend strongly on the link parameters ${ }^{(6)(7)}$. The inner speed control loop is expressed by the transfer function,

$$
\frac{\omega_{M}}{\omega^{* c m d}}=\frac{K_{P}(s+\beta)\left(b_{2} s^{2}+b_{1} s+b_{0}\right)}{\left(s+\alpha_{1}\right)\left(s+\alpha_{2}\right)\left(s+\alpha_{3}\right)\left(s+\alpha_{4}\right)} \ldots \ldots
$$

The feedforward controller are designed to eliminate the dominant poles, $\alpha_{1}$ and $\alpha_{2}$. The unity steady state gain of the feedforward controller is guaranteed by the designated gain $K_{F}$. The transfer function of this feedforward controller is expressed by,

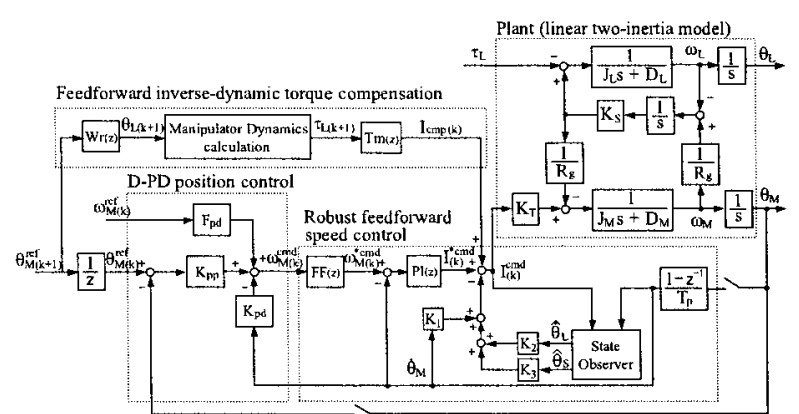

Fig. 9. Proposed robust control scheme (Controller2) for motion control system

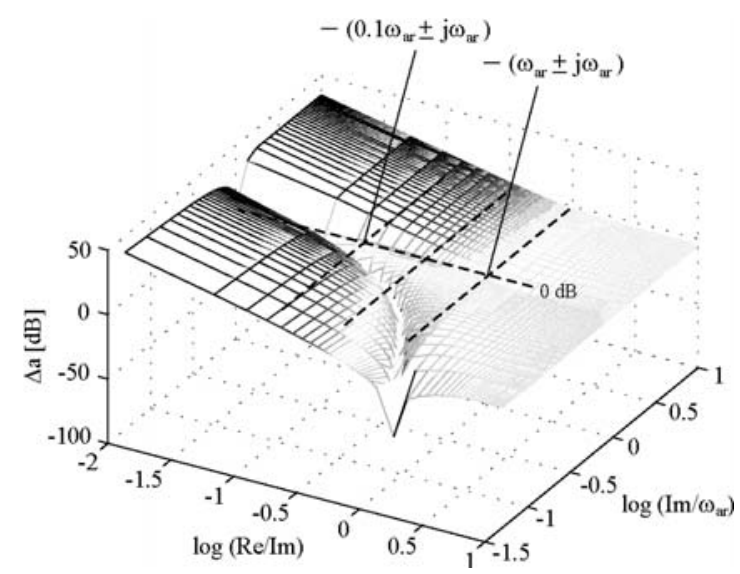

Fig. 10. Robust pole location with pole fluctuation caused by inertia variation

$$
F F(s)=\frac{K_{F}\left(s+\alpha_{1}\right)\left(s+\alpha_{2}\right)}{(s+\beta)\left(s+\alpha_{5}\right)} .
$$

Since the dominant poles are strongly depend on link parameters, such as $J_{L}$ and $D_{L}$, the fluctuation of these assigned poles occurs by the attitude change during robot movement. The inertia moment of each joint changes during robot movement and may bring the system to undesired response. In Controller 2 scheme, the robust pole allocation against the inertia variation for two dominant complex conjugate poles $-(R e \pm j I m)$ is considered.

The pole fluctuation from inertia variation to the maximum value $J_{\text {Lmax }}$, which is set at five times of the nominal value $J_{L}$, and robust pole locations of one joint of the tested manipulator are shown in a three dimensional plot in Fig. 10.

The abscissas are coordinates representing the relationship of the complex poles and the two-inertia parameters. The logarithmic values of ratio $(\mathrm{Re} / \mathrm{Im})$ and $\left(\mathrm{Im} / \omega_{a r}\right)$ for the designated pole-pair are used for the coordinates. The ordinate represents the damping ratio between the designated pole $\zeta_{\text {designated }}$ and the fluctuated value $\zeta_{\text {fluctuated }}$ which occurs from inertia fluctuation. This ratio is expressed in decibel unit as shown in Eq. 10. It is obvious that if this ratio is approaching zero, the pole fluctuation against inertia variation is small. Hence, the robust pole locations are on the intersections of the 0dB-line and the pole fluctuation graph as shown in Fig. 10.

$$
\Delta a=20 \log \left(\frac{\zeta_{\text {fluctuated }}}{\zeta_{\text {designated }}}\right)
$$

For the experimental setup robot, this paper points out that 
the robust dominant conjugate poles should be allocated on the straight line from $-\left(0.1 \omega_{a r} \pm j \omega_{a r}\right)$ to $-\left(\omega_{a r} \pm j \omega_{a r}\right)$.

\subsection{Feedforward Inverse Dynamic Compensation}

Coupled torque and gravity torque have to be compensated to achieve the linearized two-inertia model. The feedforward inverse-dynamic torque compensation is used to compute this regarded disturbance torque. The dynamic torque required for driving robot motion is already shown in Eq. 1. This dynamic torque can be separated into nominal term and nonlinear disturbance term. The nominal term consists of the torque required for nominal link inertia and viscous friction as shown in Eq. 11. The disturbance term that represents both the coupled torque from other links, the inertia variation, and the gravity force are shown in Eq. 12.

$$
\begin{aligned}
& \tau_{L n}=J_{L} \ddot{\theta}_{L}+D_{L} \dot{\theta}_{L} \ldots \ldots \ldots \ldots \ldots \ldots \ldots \\
& \tau_{L}=\tau-\tau_{L n} \\
& \quad=\left(D\left(\theta_{L}\right)-J_{L}\right) \ddot{\theta}_{L}+V\left(\theta_{L}, \dot{\theta}_{L}\right)+G\left(\theta_{L}\right) \ldots \ldots \ldots
\end{aligned}
$$

In this paper, viscous friction is considered as the friction term appeared in the robot model. The feedforward compensation is realized by applying the position reference to compute the disturbance torque and compensate into the system. In this compensation technique, the load-side position and the load-side speed are necessary to compute the dynamic torque. They are calculated from motor-side position reference $\theta_{M}^{r e f}$, by using the transfer function $\operatorname{Wr}(z)$.

$$
\begin{aligned}
W r(z) & =\frac{\theta_{L(k)}}{\theta_{M(k)}^{r e f}} \\
& =\frac{b_{w 3} z^{3}+b_{w 2} z^{2}+b_{w 1} z+b_{w 0}}{z^{4}+a_{w 3} z^{3}+a_{w 2} z^{2}+a_{w 1} z+a_{w 0}} \ldots \ldots
\end{aligned}
$$

The coefficients of numerator and denominator of this transfer function depend on parameters of the two-inertia model. The disturbance torque at the load side of each joint is computed by using the inverse dynamic equation. The computed disturbance torque from the dynamic model is then transferred to the compensation current $I_{c m p}$, by the transfer function $T^{m}(z)$. The unit time delay $\left(z^{-1}\right)$ has to be included to make it a proper transfer function.

$$
\begin{aligned}
\operatorname{Tm}(z) & =\frac{I_{c m p(k)}}{\tau_{L(k+1)}} \\
& =\frac{z^{5}+a_{t 4} z^{4}+a_{t 3} z^{3}+a_{t 2} z^{2}+a_{t 1} z+a_{t 0}}{b_{t 4} z^{5}+b_{t 3} z^{4}+b_{t 2} z^{3}+b_{t 1} z^{2}+b_{t 0} z}
\end{aligned}
$$

To implement this current compensation, the motor position reference used for the feedforward compensation is required to be one sampling-time future value to make exact current compensation to the system. In practice, the motor position reference for the main control system is delayed at one sampling time by putting in process with unit time delay as shown in Fig. 9. Then, the position reference for the feedforward compensation is relatively one sampling future value. From this technique, the inverse-dynamic torque compensation can be implemented by feedforward method.

4.3 D-PD Position Control The robust position control with straightforward design method for robot application is introduced in this paper. It consists of PD control and velocity feedforward part. PD controller gains, $K_{p p}$ and $K_{p d}$, are designed by employing robust control design approach, CDM (Coefficient Diagram Method) ${ }^{(12)(13)}$. These gains are designed by considering the stability condition and settling time of the system. Since CDM uses the closed-loop characteristic polynomial in design, the stability and response of the system can be arbitrarily designated. By using the desired value of stability index $\gamma_{1}$, and the equivalent time constant $\tau_{c}$, the characteristic of the system can be improved. In the two-inertia system, the recommended value for $\gamma_{1}$ is 2 and for $\tau_{c}$ is $0.05^{(13)}$.

$$
\begin{gathered}
\left(\begin{array}{c}
K_{p d} \\
K_{p p}
\end{array}\right)=\left(\begin{array}{c}
\alpha_{3} \alpha_{4} \alpha_{5} J_{L} \\
\alpha_{3} \alpha_{4} \alpha_{5} J_{L}\left(1-\left(D_{L} \gamma_{1} /\left(K_{S} \tau_{c}\right)\right)\right. \\
\alpha_{3} \alpha_{4} \alpha_{5} J_{L}\left(D_{L}-K_{S} \tau_{c}\right) / K_{S} \\
\alpha_{3} \alpha_{4} \alpha_{5} J_{L}\left(D_{L}-J_{L} \gamma_{1} / \tau_{c}\right) / K_{S}
\end{array}\right)^{-1} \\
\left(\begin{array}{c}
-\alpha_{3} \alpha_{4} \alpha_{5} J_{L n} \\
-\alpha_{3} \alpha_{4} \alpha_{5} J_{L n}+J_{L} \gamma_{1}\left(\alpha_{3} \alpha_{4}+\alpha_{4} \alpha_{5}+\alpha_{3} \alpha_{5}\right) / \tau_{c}
\end{array}\right)
\end{gathered}
$$

To improve the transient position response and reduce the state error of the position control, the feedforward gain $F_{p d}$, is determined by a zero assignment based on the minimumphase condition. The transfer function of position control system is then

$$
\frac{\theta_{M}}{\theta_{M}^{r e f}}=\frac{K_{P} K_{F}\left(K_{p p}+s F_{p d}\right)\left(b_{2} s^{2}+b_{1} s+b_{0}\right)}{s^{4}+a_{n 3} s^{3}+a_{n 2} s^{2}+a_{n 1} s+a_{n 0}} \ldots
$$

\section{Experimental Results}

The experimental results with comparison of the control performance between the conventional Controllerl and the proposed Controller 2 scheme are inspected. The nominal parameters used in controller design are also compared between the conventional identification method and the proposed identification method.

The Controller 2 scheme shows the considerable improvement in tracking response based on parameters from both conventional and proposed identification method. The experimental results are shown in Fig. 11 for low speed motion

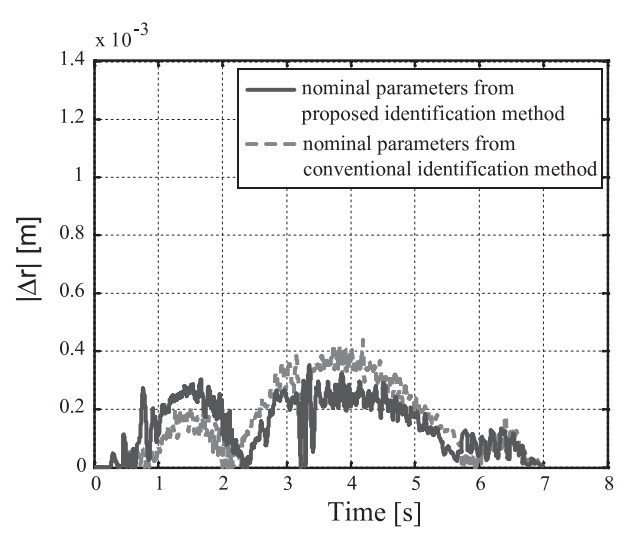

Fig. 11. Comparison of low-speed tracking path errors in robust control scheme 


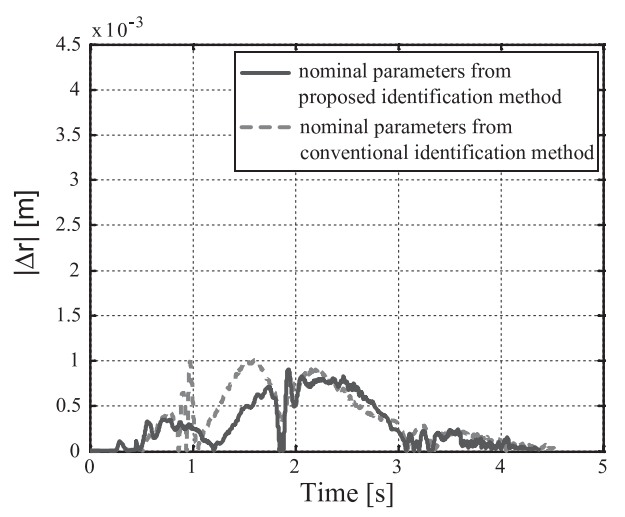

Fig. 12. Comparison of high-speed tracking path errors in robust control scheme

Table 3. Maximum tracking error and area of error from experimental results of Controller1

\begin{tabular}{c|c|c|c|c}
\hline \hline \multirow{2}{*}{$\begin{array}{c}\text { Motion } \\
\text { Speed }\end{array}$} & \multicolumn{2}{|c|}{ Maximum error (mm) } & \multicolumn{2}{c}{ Area of error (mm-s) } \\
\cline { 2 - 5 } & $\begin{array}{c}\text { Conventional } \\
\text { Identification } \\
\text { Method }\end{array}$ & $\begin{array}{c}\text { Proposed } \\
\text { Identification } \\
\text { Method }\end{array}$ & $\begin{array}{c}\text { Conventional } \\
\text { Identification } \\
\text { Method }\end{array}$ & $\begin{array}{c}\text { Proposed } \\
\text { Identification } \\
\text { Method }\end{array}$ \\
\hline Low & 1.25 & 0.87 & 2.83 & 1.97 \\
High & 2.91 & 2.41 & 4.22 & 3.51 \\
\hline \hline
\end{tabular}

Table 4. Maximum tracking error and area of error from experimental results of Controller2

\begin{tabular}{c|c|c|c|c}
\hline \hline \multirow{3}{*}{$\begin{array}{c}\text { Motion } \\
\text { Speed }\end{array}$} & \multicolumn{2}{|c|}{ Maximum error (mm) } & \multicolumn{2}{c}{ Area of error (mm-s) } \\
\cline { 2 - 5 } & $\begin{array}{c}\text { Conventional } \\
\text { Identification } \\
\text { Method }\end{array}$ & $\begin{array}{c}\text { Proposed } \\
\text { Identification } \\
\text { Method }\end{array}$ & $\begin{array}{c}\text { Conventional } \\
\text { Identification } \\
\text { Method }\end{array}$ & $\begin{array}{c}\text { Proposed } \\
\text { Identification } \\
\text { Method }\end{array}$ \\
\hline Low & 0.44 & 0.33 & 1.23 & 1.12 \\
High & 1.01 & 0.91 & 1.71 & 1.52 \\
\hline \hline
\end{tabular}

and in Fig. 12 for high speed motion.

The maximum value of tracking error and the area of tracking error are calculated from the experimental data. These values are used as an index in performance comparison between the conventional and the proposed parameter identification method as well as the both control schemes. Table 3 and Table 4 show the maximum and the area of tracking error from experimental data of Controller1 and Controller2 scheme, respectively.

From the results in Fig. 7 and 8, the tracking control with Controllerl scheme shows that the tracking error is apparently reduced when the parameters from the new identification method are employed. In the Controller 2 scheme, the same results are also noticeable in Fig. 11 and 12; however, the difference in tracking path error is not obvious as in the case of Controllerl. Hence, the Controller 2 scheme gains the robust characteristic against the nominal parameter variation in two-inertia model.

\section{Conclusion}

The new nominal parameter identification method based on frequency domain was proposed. Inertia of robot link and elastic constant of gear drive are related to the frequency response of the open loop two-inertia system. Improvement in the performance of the experimental results affirms the effectiveness of the identified parameters. For conventional control scheme (Controller1) with the proposed parameter identification method, the performance of the control system improves up to $30 \%$ for low speed motion and up to $17 \%$ for high speed motion.

In addition, the proposed robust control scheme (Controller2) proves its performance on the parameter variation. The experimental results confirmed the robust performance when using both parameters from the conventional and the proposed identification method. The robust control scheme has an advantage to implementing on the highly nonlinear system such as a robot manipulator.

(Manuscript received July 2, 2004)

\section{References}

( 1 ) P.S. Gandhi and F.H. Ghorbel: "Closed-Loop Compensation of Kinematic Error in Harmonic Drives for Precision Control Applications", IEEE Trans. Control Systems Technology, Vol.10, pp.759-768 (2002-11)

( 2 ) C. Abdallah, D. Dawson, P. Dorato, and M. Jamshidi: "Survey of Robust Control for Rigid Robots", IEEE Control Systems Magazine, Vol.11, pp.2430 (1991-2)

( 3 ) M.W. Spong: "Modeling and Control of Elastic Joint Robots", ASME J. of Dynamic Systems, Measurement, and Control, Vol.109, pp.310-319 (198712)

( 4 ) P. Tomei: "A Simple PD Controller for Robots with Elastic Joints", IEEE Trans. on Automatic Control, Vol.36, pp.1208-1213 (1991-10)

( 5 ) T. Miyazaki and K. Ohishi: "Robust Speed Control System Considering Vibration Suppression Caused by Angular Transmission Error of Planetary Gear", IEEE/ASME Trans. on Mechatronics, Vol.7, pp.235-244 (2002-6)

( 6 ) T. Miyazaki, S. Otaki, S. Tungpataratanawong, and K. Ohishi: "High Speed Motion Control Method of Industrial Robot Based on Dynamic Torque Compensation and Two-Degrees-of-Freedom Control System", T. IEEJ, Vol.123D, No.5, pp.525-532, (2003-5) (in Japanese)

( 7 ) S. Tungpataratanawong, K. Ohishi, and T. Miyazaki: "Robust Motion Control of Industrial Robot Manipulator Based on Feedforward Torque Compensation and D-PD Control", in Proceedings of the 6th Japan-France and 4th Asia-Europe Mechatronics Congress, pp.432-437, Japan (2003)

( 8 ) T. Mita and K. Osuka: "Introduction to Robot Control", Corona Publishing, Tokyo, pp.81-91 (1989)

( 9 ) C.G. Atkeson, C.H. An, and J.M. Hollerbach: "Estimation of inertial parameters of manipulator loads and links", Int. J. Robotics Res., Vol.5, pp.101-119 (1986)

(10) G. Calafiore, M. Indri, and B. Bona: "Robot Dynamic Calibration: Optimal Excitation Trajectories and Experimental Parameter Estimation", J. Robotic Systems, Vol.18, pp.55-68 (2001-2)

(11) J.J. Craig: "Introduction to Robotics: mechanics and Control", 2nd ed., Reading, MA: Addison-Wesley (1989)

(12) S. Manabe: "The Application of Coefficient Diagram Method to ACC Benchmark Problem", in Proceedings of the 2nd Asian Control Conference, pp.II135-II138, Seoul (1997-7)

(13) S. Manabe: "Controller Design of Two-Mass Resonant System by Coefficient Diagram Method", T. IEEJ, Vol.118-D, No.1, pp.58-66 (1998-1) (in Japanese)

Somsawas Tungpataratanawong (Student Member) was born in

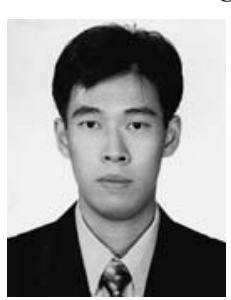
Bangkok, Thailand, on March 31, 1976. He received his B.S. degree from King Mongkut's Institute of Technology Ladkrabang, Thailand, in 1997 and M.S. degree from Nagaoka University of Technology, Japan, in 2003, all in electrical engineering. At present, he is a Ph.D. student at the Nagaoka University of Technology. His research interests include robot motion and force control, especially in application with industrial manipulator. 
Kiyoshi Ohishi (Member) received the B.S., M.S., and Ph.D. degrees,

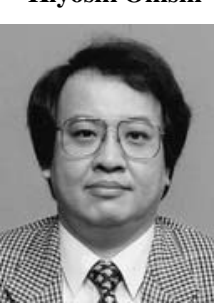
all in electrical engineering, from Keio University, Yokohama-city, Japan, in 1981, 1983, and 1986, respectively. From 1986 to 1993, he was an Associate Professor with Osaka Institute of Technology, Osakacity, Japan. From 1993 to 2003, he was an Associate Professor with Nagaoka University of Technology, Nagaoka-city, Japan. Since August 2003, he has been a Professor at the same university. Dr.Ohishi is a member of the Institute of Electrical Engineers of Japan (IEEJ), Society of Instrument and Control Engineers, the Japan Society of Mechanical Engineers, and the Robotics Society of Japan.
Toshimasa Miyazaki (Member) received the B.S., M.S., and Ph.D.

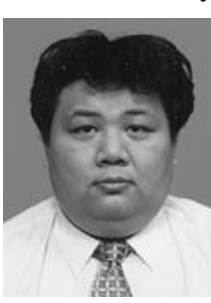
degrees, all in electronic engineering, from Nagaoka University of Technology, Nagaoka-city, Japan, in 1994, 1996, and 1999, respectively. Since 1999, he has been a Research Associate with Nagaoka National College of Technology, Nagaoka-city, Japan. His research interests include motion control and power electronics. Dr.Miyazaki is a member of the Institute of Electrical Engineers of Japan (IEEJ), the Society of Instrument and Control Engineers, and the Robotics Society of Japan. 